J. H. DE GROOT, 's Gravenhage.

J. J. M. DE GROOT, 's Gravenhage.

Jhr. c. DE giJselaAR, 's Gravenhage.

Mr. H. F. L. hamelberg, Arnhem.

J. DE Koo, Amsterdam.

H. т. KRABBE, 's Gravenhage.

J. A. KRUiJt, Djeddah.

J KUYPER HZN., 's Gravenhage.

Mr. G. G. VAN HARENCARSPEL, 's Hage. Jhr. c HaRtsen, Amsterdam.

N. D. LAMMERS VAN TOORENBURG, 's Hage.

Mr. J. W. van LANSBERge, Brummen.

Mr. H. O. VAN DER LINDEN, Dordrecht.

J. J. Hasselman, Tiel.

Mr. J. HeEMSkerk az., 's Gravenhage. Mr. A. heemskerk Az., Cä̈ro.

B. HELDRING, Amsterdam.

Mr. G. T. H. HENNY, 's Gravenhage.

Mr. J. E. HENNY, Amsterdam.

Mr. W. A. HENNY, Arnhem.

J. K. VAN DER HEIJDEN, 's Gravenhage.

F. E. HEIJNEN, Florence.

H. HIEBINK, Zutfen.

H. P. HOEVENAAR VAN GELDROP, 's Hage. N. HOFSTEDE, 's Gravenhage.

Prof. Dr. J. J. DE HOLLANDER, Breda. G. DU RIJ vaN BEest holle, 's Hage. Dr. м. тн. houtsma, Leiden.

J. HUDIG DZ., Amsterdam.

H. с. HUMмe, 's Gravenhage.

Jhr. Mr. J. HUYDECOPER VAN MAARSSEveEn, Maarsseveen.

H. A. INSINGer, Baarn.

Mr. E. H. 's $\mathrm{J}_{\mathrm{ACOB}}$, Utrecht.

P. W. Jansen, Amsterdam.

C. A. J. L. JEEKEL.

Dr. F. A. JENTINK, Leiden.

C. J. M. JONGKINDT CONINCK, Wageningen.

A. C. Joosten, Amsterdam.

Prof. Dr. A. w. т. JuYnboll, Delft.

Mr. w. van der kaAiJ, Leiden.

Prof. Dr. c. M. KAN, Amsterdam.

Prof. Dr. H. Kern, Leiden.

c. E. tan kesteren, 's Gravenhage.

Mr. L. w. c. KeUchenius, 's Hage.

w. F. VAN ERP TAALMAN KIP, 'sHage.

H. C. KLINKert, Leiden.

J. H. P. Е. KNIPHORST, 's Gravenhage.

E. H. LASONDER, Utrecht.

Prof. Mr. P. A. VAN DER LITH, Leiden. s. LOCKER DE bRUIJNe, 's Gravenhage.

Mr. H. HOPE LOUDON, 's Gravenhage.

Prof. Mr. J. DE LOUTER, Utrecht.

D. MaARSCHALK, 's Gravenhage.

Mr. J. C. DE Marez oyens, 's Gravenhage.

Prof. Dr. к. martin, Leiden.

Dr. в. F. мatthes, 's Gravenhage.

Mr. w. c. MeEs, Amsterdam.

Mr. F. ALting mees, 's Gravenhage.

Prof. J. J. Meinsma, Delft.

J. w. MIDDElbuRG, 's Gravenhage.

L. J. J. MichIELSEN, 's Gravenhage.

J. MILLARD, 's Gravenhage.

Mr. c. J. F. Mirandolle, Haarlem.

J. F. ғ. мовт, 's Gravenhage.

Dr. к. w. м. MontisN, Schiedam.

H. F. MoRbotter, 's Hage.

H. MULLER sZn., Rotterdam.

A. D. van der gon netscher, 's Hage.

P. M. NETSCHER, 's Gravenhage.

Prof. G. K. niemann, Delft.

Jhr. Mr. o. E. van NISPEn, 's Hage.

M. NIJHOFF, 's Gravenhage.

Mr. o. W. STAR NUMAN, 's Gravenhage.

A. P. M. VAN OORDT, Leiden.

H. J. oosting, Assen.

Mr. r. T. PAHUd de mortanges, Arnhem.

w. PAHUd de mortanges, 's Hage.

Jhr. Mr. e. van panhuYs, 's Hage.

Mr. J. G. PATIJN, 's Gravenhage.

Mr. D. L. F. DE PAULY, Florapark,

Haarlem.

J. C. De Kock van LeEUWWen, 's Hage. M. P. PELs, Amsterdam. 
Mr. F. M. C. PELS RIJKen, Arnhem. M. T. H. PERElaEr, 's Gravenhage. Prof. Mr. N. G. PIERSON, Amsterdam. Prof. Dr. J. piJnappel gzn., Leiden. w. PLeyte, Leiden.

Dr. FRANçOIS P. L. POLLen, Scheveningen. A. POMPE, Breda.

Jhr. . POMPE VAN MEERDERVOORT, 's Gravenhage.

v. w. posthumus, Amsterdam.

a. pRUYS VAN Der hoeven, 's Hage.

A. S. fRansen VAN de PUtTe, 's Hage. Jhr. Mr. J. K. W. QUARLES VAN UFFord, 's Gravenhage.

Jhr. Mr. w. van Rappard, 's Hage.

M. A. VAN RHEDE VAN DER KLOOT, 's Gravenhage.

Jhr. Mr. G. C. J. van Reenen, 's Hage. o. VAN REES, 's Gravenhage,

J. G. F. RIEDEL, Utrecht.

Dr. w. N. DU RIEU, Leiden.

Jhr. Mr. J. ROËLL, 's Gravenhage.

J. W. ROSKEs, Rotterdam.

c. RUEB cz., Rotterdam.

Dr. E. VAN RIJCKEVORSEL, Rotterdam. R. G. DE SEYFF, Genève.

w. Schagen van LeEUWEN, Delft.

Mr. J. c. J. vAN DER SCHALK, NoordwijkBinnen.

D. scheltema, Haarlem.

Prof. Dr. G. SChlegel, Leiden.

Prof. H. SCHLEgel, Leiden.

J. F. L. SCenNEIDER, Delft.

Dr. J. sEMmeLINK, 's Gravenhage.

Mr. L. SERRURIER, Leiden.

a. M. Servatius, Terwolde bij Deventer.

Mr. C. J. sICKesz, Laren.

Jhr. Mr. w. sIx, 's Gravenhage.

Jhr. J. D. sIX, 's Gravenhage.

Mr. L. A. J. W. Baron SLOET VAN DE BEELE, Arnhem.

Dr. H. SMeding, Haarlem.
JOH. F. SNELLEMAN, Rotterdam.

Ur. C. SNOUCK hURGRonJe, Leiden.

Prof. J. SPANJAARD, Delft.

Dr. J. s. SPEIJER, Amsterdam.

J. P. SPRENGer VAN EIJK, 's Gravenhage.

Dr. N. P. VAN DER stoK, Rijswijk

(Buitengedachten).

Jhr. Mr. v. DE stuers, 's Gravenhage. Jhr. c. A. van sypesteisn, 's Gravenhage. A. W. sisthоғт, Leiden.

Mr. J. P. R. TAK VAN POORTVLIET, 's Hage. P. e. tegelberg, Amsterdam.

Mr. G. van tienhoven, Amsterdam. N. TRAKRANEN, Amsterdam.

Prof. Dr. J. J. P. VALETON, Groningen. Mr. s. A. vening meInesz, Rotterdam. H. VERPLOEGH, 's Gravenhage.

D. D. VETH, Amsterdam.

T. P. VIRUly, Leiden.

c. A. M. VAN VLIET, 's Gravenhage.

Dr. J. VAN DER vLIET, Haarlem.

B. R. F. VAN VLIJMEN, Nijmegen.

J. DE VOGEL, 's Gravenhage.

JOOST VAN volLENHOVEN, Rotterdam.

Mr. J. A. G. Baron DE vos VAN STEENWIJK, Utrecht.

Prof. A. C. VREEDE, Leiden.

E. DE WAAL, 's Gravenhage.

W. A. VAN WALCHEREN, 's Gravenhage.

Dr. s. J. WARREN, Dordrecht.

A. E. WASZEKLEWICZ, 's Gravenhage.

A. W. P. WEITZEL, 's Gravenhage.

G. P. Westerman, Amsterdam.

c. F. W. WICHERS VAN KERCHEM, Leiden.

Prof. Dr. c. E. A. WICHManN, Utrecht.

WILLEM WIJT, Rotterdam.

G. A. WILKEN, Leiden.

J. WOLBERS, Utrecht.

R. ARENDSEN DE WOLFF, 's Gravenhage.

D. G. E. WOLTERBEEK MULLER, Voorourg.

J. wüste, Amsterdam. 
Jhr. Mr. H. C. VAN DER WIJCK, 's Hage. Dr. TH. CH. L. WiJnMalen, 's Hage. Jhr. Mr. H. van Der WIJCK, 's Hage. s. B. zeverijn, Amsterdam.

\section{CORRESPONDEERENDE LEDEN.}

C. H. B. VON ROSENBerg, 's Gravenhage.

P. A. TIELE, Utrecht.

J. c. neurdentburg, Rotterdam.

c. A. de Magnin, Parijs.

\section{NEDERLANDSCH OOST-INDIË.}

\section{DONATEUR.}

F. 's ЈАсов, Gouverneur-Generaal van Ned.-Indië, Batavia.

\section{GEWONE LEDEN.}

G. C. D’ABo, Landhuurder, Batoe Djamoes (Soerakarta).

J. w. H. ADÈr, Predikant te Batavia.

J. A. aEckerin, Secretaris van de residentie Benkoelen (Sumatra).

J. E. ALBrecht, President van de Weeskamer, Bibliothecaris v. h. Bataviaasch Genootschap van Kunsten en Wetenschappen, Batavia.

Jhr. ғ. H. P. van ALPHeN, Kommandant van de Zeemacht in N.-I., Batavia. H. м. andrée wiltens, Vice-president van den Raad van Ned.-Indië, Batavia. Mr. N. P. van Den berg, President van de Javasche Bank, Batavia Mr. L. w. c. van den Berg, Ambtenaar voor de Inlandsehe talen, Batavia. J. BoDDÉ, Zendeling te Tengah-Sonder (Menado).

E. воSCH, Assistent-resident voor de policie, Padang (Sumatra's Westkust).

J. A. H. BReymans, controleur te Bauding Agoeng (Palembang).

w. p. van charante, President viln het Liefdadigheids-gesticht enz. te Depok (Buitenzorg).

Mr. J. A. VAN DER CHIJs, Ambtenaar belast met het toezicht over 't archief, Batavia.

Mr. J. W. F. Cohen stuart, Advocaat, Semarang.

A. J. W. van DELDEN, Directeur van de Spaarbank, Batavia.

J. A. DEzentJe, Landhuurder, Ampal, residentie Soerakarta. 
s. VAN DISSEL, Inspecteur van het inlandsch onderwijs, Batavia.

P. VAN DIJK, Hoofdmijningenieur, Batavia.

Mr. J. W. ESSERS, Raadsheer in het Hooggerechtshof, Batavia.

1. FOCKENS JR., Controleur 2e klasse, Krawang.

D. Gerth VAN WIJK, Leeraar in de Maleische taal, Commissaris van het Instituut, Batavia.

Di. J. H. F. SOLLEWYN GELPKE, Hoofd-inspecteur der kultures, Baitenzorg.

G. P. H. H. GONGGRIJP, Batavia.

w. P. Groeneveldt, Secretaris van het Dep. onderwijs enz., Batavia.

N. GRAafland, Directeur der Kweekschool, Tanawangko (Menado).

Dr. J. G. H. GUNning, Ambtenaar voor de inlandsche talen, Soerakarta,

Dr. C. GutTeling, Geneesheer, Batavia.

Mr. J. A. нAAKMAN, Redacteur Bat. Handelsblad, Batavia.

J. наввема, Hoof́̃onderwijzer, Amboina.

L. K. Harmsen, Hoofdonderwijzer te Padang Sidempoean, Tapanoeli.

G. J. harreboméE, Controleur le klasse te Kotan Nopan (afd. Rau, Sumaira's Westkust).

S. E. HARTHOORN, Leeraar in de Indische taal-, land-en volkenkunde, Batavia.

A. L. van hasselt, Referendaris Algemeene Secrétarie, Commissaris van het lnstitunt, Batavia.

H. van heuckeldm, Assist.-resident te Padang Sidempoean (Sumatra's Westkust).

J. heijting, Resident van Kadoe, Magelang.

Dr. P. heisting, Predikant te Poerworedjo.

в. hoztink, Tolk voor de Chineesche taal te Deli Médan (Sumatra's Oostkust).

G. w. w. c. Baron van hoevell, Controleur le klasse te Kajoetanam, afd.

Priaman (Sumatra's Westkust).

w. Hовzoo, Zendeling, Semarang.

Dr. J. w. hopmann, Geneesheer, Buitenzorg.

K. F. HOLLe, Adviseur, Waspada (Preanger Regentschappen).

Jhr. H. W. F. HORA SICCAMA, Resident, Batavia.

Dr. D. w. HoRst, Controleur le klasse, te Kroë, resid. Benkoelen.

P. A. Jansz, Zendeling-leeraar, Depok.

Mr. т. H. Der Kinderen, Lid van den Raad van Ned.-Indië, Batavia.

J. knebel, Controleur le klasse, Pasoeroean.

Jhr. ж. н. ш. DE коск, Secretaris van de Padangsche Bovenlanden, Fort de Kock.

G. P. J. VAN KOETSvelD, Secretaris voor de inlaudsche zaken, Makassar.

P. J. Kooreman, Controleur le klasse, tijdelijk te Batavia.

G. J. VAN KOOTEN, le Luitenant artillerie N. I., Batavia.

J. Kreemer, Zendeling-leeraar, Malang (Pasoeroean).

R. C. Kroesen, Resident van Bengkalis, Oostkust van Sumatra.

L. CH. KRUIJFF, Notaris, Probolinggo

J. c. Kummer, Adjunct-inspecteur van het inlandsch onderwijs, Magelang. 
Mr. н. кuneman, Chef der afdeeling Statistiek ter Algemeene Secretarie, Batavia.

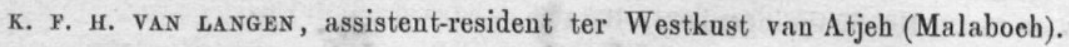
c. H. M. LERoux, Controleur le klasse te Medan, afd. Deli (Oostkust van Sumatra). Mr. H. D. LEvyssohn norman, Lid van den Raad van Indie, Batavia.

Dr. w. van lingen, Predikant te Pekalongan.

H. L. C. TE MECHELen, Assistent-resident van Djoewana, Japara.

J. J. NAEFr, Controleur le klasse, Sipirok (Sumatra's Westkust).

c. van der GoN Netscher, Assistent-resident, Panaroekan (Besoeki).

J. H. PANNEKOEK, Algemeene Secretaris, Buitenzorg.

Mr. M. C. PIEPERS, Raadsheer in het Hoog-Gerechtshof, Batavia.

c. POENSEN, Zendeling-leeraar, Kedirie.

H. E. PRINS, Secretaris van het Gouvernement Sumatra's Wesstkust, te Padang.

RADEN ADIPATI ARIA KOESOEMa DININGRAT, Galoeh.

RADEN ADIPATI ARIA SOERIA dI REDJA, Cheribon.

RADEN ADIPATI sosRo NEGORO, Rijksbestuurder van Soerakarta.

RADEN MAS ADIPATI ARIO TJONDRONEGORO, Brebes.

RADEN MAS ISMANGOEN DANOE WINOTO, Adjunct-inspecteur van het Inlandsch onderwijs, Probolinggo.

RADEN mas tommenggoeng pandji adiningrat, Regent van Demak.

H. A. P. H. RAvenswaAiJ, Chef der boekhandelaarsfirma G. C. T. van Dorp \& Co., Semarang.

Mr. F. G. A. REITZ, President van den Landraad te Ngawi (residentie Madioen). Dr. H. M. D. VAN RIEMSDJJK, Fort de Kock.

Dr. L. W. G. DE Roo, Inspecteur van Financiën, Batavia.

J. м. Rоsкорғ, Inspecteur van het lager onderwijs, Semarang.

B. J. E. Rозкотт, Assistent-resident Tebing Tinggi (Palembang).

L. w. тн. sснмірт, Hoofdonderwijzer, Makassar.

K. L. van schouwenburg, Directeur Hoogere Burgerschool, Semarang.

L. $\boldsymbol{P}$. teiJl schuitemaker, Hoofdonderwijzer, ain de kweekschool te Probolinggo.

J. F. н. schultz, Controleur le klasse, Manna, res. Benkoelen.

W. H. SENN VAN BASEL, Serang (Bantam)

B. smissaert, Assistent-resident van Sidajoe (Soerabaja).

A. sol, Resident te Atjeh.

A. J. SPAAN, Resident te Cheribon.

Mr. w. Stortenbeker JR., Directeur van het Dep. van Onderwijs, Nijverheid en Eeredienst, Batavia.

H. N. Stuart, Tolk voor de Chineesche taal, Makassar.

Mr. J. E. DE stURler, Ambtenaar ter Algemeene Secretarie, Batavia.

P. F, laging tobias, Gouverneur van Atjeh.

J. L. VAN DER toorn, Hoofdonderwijzer, Fort de Kock (Padangsche Bovenlanden).

r. TwIss, Controleur te Sidjoendjoeng (Sumatra's Westkust). 
R. D. M. verbeek, Ingenieur le klasse, Buitenzorg.

Mr. A. w. c. verweis, President van den landraad, Batavia.

J. M. VAN vLeuten, Resident van de Preanger-Regentschappen (Bandong).

J. Rulemann voget, Predikant, Batavia.

J. H. G. vosmaer, Assistent-resident van Blinjoe, Bangka.

A. F. VON DE WALL, Batavia.

c. W. J. Wenneker, Pastoor, Medan (Deli).

J. A. B. WISELIUS, Assistent-resident voor de politie, Semarang.

H. witbols feugen, Controleur le klasse, Karimon (Riouw). 